\title{
Erratum
}

\section{Erratum to: Modelling the effect of ribosome mobility on the rate of protein synthesis}

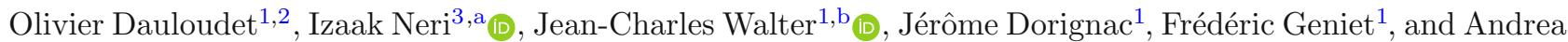
Parmeggiani ${ }^{1,2, \mathrm{c}} \mathbb{B}$

${ }^{1}$ Laboratoire Charles Coulomb (L2C), CNRS, Montpellier University, Montpellier, France

${ }^{2}$ Laboratory of Parasite Host Interactions (LPHI), CNRS, Montpellier University, Montpellier, France

${ }^{3}$ Department of Mathematics, King's College London, Strand, London WC2R 2LS, UK

Published online 26 April 2021

() EDP Sciences, SIF and Springer-Verlag GmbH Germany, part of Springer Nature 2021

Erratum to: Eur. Phys. J. E (2021) 44:19

https://doi.org/10.1140/epje/s10189-021-00019-8

In the original publication of the article were mistakes

in equations (18) and (20).

The original article has been corrected.

The original article can be found online at https://doi.org/ 10.1140/epje/s10189-021-00019-8.

\footnotetext{
${ }^{a}$ e-mail: izaak.neri@kcl.ac.uk (corresponding author)

b e-mail: jean-charles.walter@umontpellier.fr

c e-mail: andrea.parmeggiani@umontpellier.fr
} 